\title{
Exercise capacity in adult patients with congenitally corrected transposition of the great arteries
}

\author{
P M Fredriksen, A Chen, G Veldtman, S Hechter, J Therrien, G Webb
}

\begin{abstract}
Objective-To examine cardiopulmonary values, static lung function, and ejection fraction in adult patients with congenitally corrected transposition of the great arteries (CCTGA).

Patients and methods-41 patients who had undergone static lung function testing and cardiopulmonary exercise tests with measurements of ejection fraction were identified at the Toronto Congenital Cardiac Centre for Adults.

Results-Aerobic capacity in patients with CCTGA was severely diminished, varying from $30-50 \%$ of the results achieved by healthy subjects. Normal values of right ventricular and left ventricular ejection fraction were found. However, the systemic right ventricular ejection fraction increased by $2 \%$ from rest to exercise, as opposed to the expected $>5 \%$ increase in a healthy population. The pulmonary left ventricular ejection fraction decreased by $2 \%$ at peak exercise. Conclusion-Diminished values of heart rate, forced expiratory volume in one second $\left(\mathrm{FEV}_{1}\right)$, forced vital capacity, and systolic blood pressure compared to the predicted values may contribute to the reduced maximal oxygen uptake $\left(\mathrm{VO}_{2} \max \right)$ found in patients with CCTGA. In addition, a limited increase in systolic right ventricular ejection fraction and a decrease in pulmonary left ventricle contractility suggest a dysfunction of both ventricles.

(Heart 2001;85:191-195)
\end{abstract}

Keywords: aerobic capacity; ventricular dysfunction; lung function; congenitally corrected transposition of the great arteries

Congenitally corrected transposition of the great arteries (CCTGA) is a rare congenital disease first described by Von Rokitansky in 1875. ${ }^{1}$ CCTGA (L-transposition; atrioventricular discordance and ventriculoarterial discordance $)^{2}$ has been extensively described previously. ${ }^{3-5}$ Common associated anomalies include ventricular septal defect (VSD), pulmonary stenosis, and left atrioventricular (morphological tricuspid valve) valve regurgitation, as well as complete heart block. ${ }^{2}$

A major concern in patients with CCTGA is whether their right ventricle is able to support systemic pressure over many years. Previous studies have reported an increased incidence of congestive heart failure and deaths in patients with the morphologic right ventricle serving as their systemic ventricle. ${ }^{67}$ Resting ejection fraction of the systemic right ventricle has previously been reported to be normal in CCTGA and Mustard patients, for both children and adults. ${ }^{89}$ In addition, a normal contractile response to exercise has been shown. ${ }^{8}$ Ventricular dysfunction, however, may not be evident in the resting state. ${ }^{9}$ Measurements of maximal oxygen uptake $\left(\mathrm{VO}_{2} \mathrm{max}\right)$ would therefore be useful to help understand exercise performance. Information on $\mathrm{VO}_{2} \mathrm{max}$ and static lung function in adult patients with CCTGA is currently absent.

The aim of the present study is to examine ventricular function at rest and at exercise as well as static lung function in adult patients with CCTGA, and to compare these results to healthy subjects.

\section{Patients and methods}

PATIENT POPULATION

In this retrospective study we found 131 patients with CCTGA listed in the database at the Toronto Congenital Cardiac Centre for Adults (TCCCA) with late mortality ( $>18$ years) of 28 patients $(21.4 \%)$. Of these patients, 25 died of cardiac related incidents and three of non-cardiac incidents. Their median age of death was 33 years (range 20-61 years). Seventeen (13.0\%) patients were excluded because they underwent a Fontan procedure. Other exclusions include one patient with a heart transplant and one patient with heart and lung transplant. Of the remaining 84 patients, $41(48.8 \%)$ (19 males and 22 females), with a median age of 31 years (range 19-55 years), had performed a static lung function test, a cardiopulmonary exercise test or both. Pulmonary left ventricular ejection fraction was measured at rest and during exercise in 17 and 14 patients, respectively. Systemic right ventricular ejection fraction was measured in 21 patients at rest and 19 patients at peak exercise. The tests were conducted over a 14 year period between 1985 and 1999, with the majority of tests done in 1998-99 (56.3\%). The patients were divided into four subgroups depending on their associated defects as shown in table 1.

A pacemaker was implanted in 10 patients $(24.4 \%)$ for complete heart block. Eight patients had atrioventricular (AV) sequential pacing and two had VVI pacing. Five out of the 10 patients had good chronotropic response to exercise and did not need rate-responsiveness. 
Table 1 Patients with congenitally corrected transposition of the great arteries (CCTGA) divided into four subgroups based on associated defects

\begin{tabular}{lrl}
\hline Type & $n$ & Associated defects \\
\hline Repaired complex CCTGA & 14 & VSD repair and LV-PA conduits \\
Repaired CCTGA with TVR & 15 & With and without VSD (n=1) and/or PS $(\mathrm{n}=5)$ \\
Complex CCTGA & 10 & VSD $(\mathrm{n}=4), \mathrm{PS}(\mathrm{n}=5)$ and/or coarctation $(\mathrm{n}=1)$ \\
Simple CCTGA & 2 & Without associated defects
\end{tabular}

VSD, ventricular septal defect; LV, left ventricle; PA, pulmonary atresia; TVR, tricuspid valve replacement; PS, pulmonic stenosis.

Twenty seven patients $(65.8 \%)$ were in sinus rhythm and four patients $(9.8 \%)$ had atrial fibrillation. Fourteen patients $(34.2 \%)$ used angiotensin converting enzyme (ACE) inhibitors which were given because of deteriorating systemic ventricular function or documented episodes of systemic ventricular failure. To estimate the effect of surgery, the patients were divided into a surgery group (29 patients, $70.7 \%$ ) and a non-surgery group (12 patients, $29.3 \%$ ). The patients without surgery did not have any significant residual left to right or right to left shunts. Patients with a conduit between the pulmonary left ventricle and the pulmonary artery (18 patients, 43.9\%) were also analysed in comparison to patients without a conduit (23 patients, $56.1 \%$ ). Some of the patients with conduits had residual obstruction assessed by two dimensional transthoracic echocardiogram with Doppler. Four patients had a gradient less than $20 \mathrm{~mm} \mathrm{Hg}$, eight patients had a mild obstruction (20$40 \mathrm{~mm} \mathrm{Hg}$ ), two had moderate obstruction $(40-80 \mathrm{~mm} \mathrm{Hg}$ ), and none had severe obstruction $(>80 \mathrm{~mm} \mathrm{Hg})$.

\section{CARDIOPULMONARY TEST}

Cardiopulmonary tests were performed at Toronto General Hospital on an ergometer cycle (Elema, Sweden). Continuous measurements of expired gas values were analysed every 30 seconds (Amtech $\mathrm{VO}_{2}$ Oxygen Analyzer $\mathrm{S}-3 \mathrm{~A} / \mathrm{I})$. The analyser was calibrated before testing using known values of oxygen and carbon dioxide. During the test, measurements of blood pressure, heart rate, ventilation, and oxygen saturation $\left(\mathrm{SaO}_{2}\right)$ were taken continuously. All patients started at $20 \mathrm{~W}$ for warm-up in the first minute. Workload was increased by $20 \mathrm{~W}$ every two minutes until the patient experienced unacceptable fatigue or other symptoms. The American College of Sport Medicine guidelines for ending an exercise test were followed. ${ }^{10}$ Before the exercise tests, spirometry (SensorMedics, USA) tests were conducted according to American Thoracic Society standards with measurements of forced expiratory volume in one second $\left(\mathrm{FEV}_{1}\right)$ and forced vital capacity (FVC). ${ }^{11}$ Measurements of ejection fraction were performed using radiopharmaceutical $750 \mathrm{MBq}$ 99mTco4, which was injected before exercise.

The results of $\mathrm{VO}_{2} \max$ from the CCTGA patients were compared with a healthy population in Canada over different age groups. ${ }^{12}$ Results of $\mathrm{FEV}_{1}$ and FVC were compared to predicted values calculated from equations set out by Jones. ${ }^{13}$

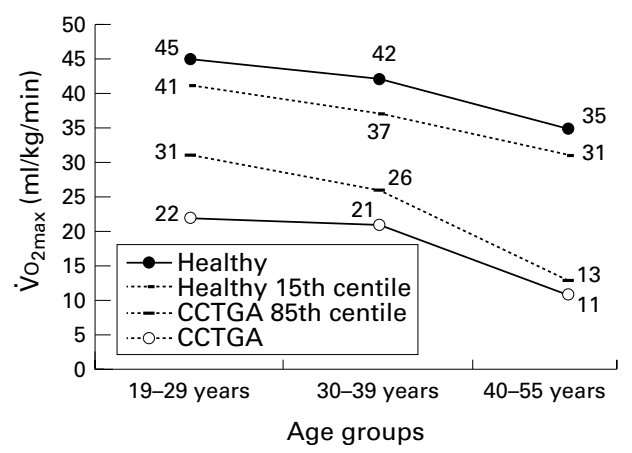

Figure 1 Compared to median values of healthy subjects (both sexes) (13) patients with CCTGA show a diminished maximal oxygen uptake (VO, max) over all age groups.

\section{STATISTICS}

Data were analysed using SPSS 9.0 for Windows. Median values with range were used descriptively to compare $\mathrm{VO}_{2} \max$ in patients with CCTGA to a healthy population. Differences in the FVC and $\mathrm{FEV}_{1}$ between CCTGA patients and predicted values were analysed with the Student $t$ test. Analysis over different age groups was done with analysis of variance (ANOVA). Multivariate linear regression modelling (stepwise backward elimination selection procedure) was used to examine the relation between age at test, type of associated defect, ACE inhibitors, sex, heart rate, type of heart rhythm, systolic blood pressure, diastolic blood pressure, surgery, conduit, FVC, and outcome variable of $\mathrm{VO}_{2} \mathrm{max}$. Pearson correlation was used to determine the relation between $\mathrm{VO}_{2} \mathrm{max}$ and maximal heart rate (HRmax). A probability value of $\mathrm{p}<0.05$ was regarded as significant.

\section{Results}

AEROBIC CAPACITY

As shown in fig 1 , aerobic capacity in patients with CCTGA was severely diminished, $\mathrm{VO}_{2} \mathrm{max}$ ranging from $11-22 \mathrm{ml} / \mathrm{kg} / \mathrm{min}$, corresponding to $30-50 \%$ of results achieved by healthy subjects. None of the patients fall within the lower 15 centile range for normal values. Multiple linear regression model showed a positive effect of FVC $(p=0.002)$, HRmax ( $\mathrm{p}<0.0001)$, and systolic blood pressure $(p=0.001)$. No effect was found with regard to age at test, sex, ACE inhibitors, associated effects, surgery, conduits or heart rhythm.

Normal values were found at rest for $\mathrm{SaO}_{2}$ (table 2). During exercise however, a decrease was displayed (table 3).

\section{HEART RATE}

A normal mean resting value was found for heart rate in the CCTGA patients, but a diminished increase with exercise was revealed, as $79 \%$ of predicted values were obtained. No differences were found between patients with pacemakers and patients in sinus rhythm.

\section{BLOOD PRESSURE}

Significantly higher values were demonstrated in males with respect to resting systolic and diastolic blood pressure (table 2). There was a 
Table 2 Resting data (mean (SD)) for patients with CCTGA. The p values refer to the differences between males and females

\begin{tabular}{llllllll}
\hline Variable & $n$ & CCTGA & $n$ & Males & $n$ & Females & $p$ Value \\
\hline SaO $_{2}$ & 24 & $96(4)$ & 13 & $96(2)$ & 11 & $96(5)$ & 0.554 \\
Heart rate (bpm) & 27 & $71(10)$ & 15 & $70(11)$ & 12 & $72(9)$ & 0.515 \\
Sys BP (mm Hg) & 37 & $116(13)$ & 18 & $121(10)$ & 19 & $111(13)$ & 0.013 \\
Dia BP (mm Hg) & 37 & $73(8)$ & 18 & $76(7)$ & 19 & $70(8)$ & 0.015 \\
MRVEF sys (\%) & 21 & $48(15)$ & 10 & $50(9)$ & 11 & $46(19)$ & 0.573 \\
MLVEF pulm (\%) & 17 & $46(19)$ & 7 & $57(15)$ & 10 & $37(17)$ & 0.026 \\
FVC obtained & 32 & $3.66(1.12)$ & 14 & $4.51(0.97)$ & 18 & $3.00(0.72)$ & $<0.0001$ \\
FVC \% predicted & 32 & $84(15)$ & 14 & $85(12)$ & 18 & $83(18)$ & 0.792 \\
FEV obtained & 32 & $2.99(0.92)$ & 14 & $3.62(0.89)$ & 18 & $2.50(0.60)$ & $<0.0001$ \\
FEV $_{1} \%$ predicted & 32 & $84(17)$ & 14 & $82(16)$ & 18 & $85(19)$ & 0.692
\end{tabular}

$\mathrm{SaO}_{2}$, oxygen saturation; Sys BP, systolic blood pressure; Dia BP, diastolic blood pressure; MRVEF sys, morphologic right ventricular ejection fraction (systemic); MLVEF pulm, morphologic left ventricular ejection fraction (pulmonary); FVC, forced vital capacity at rest, $\mathrm{FEV}_{1}$, forced expiratory volume in one second at rest.

significant increase in systolic and diastolic blood pressure from rest to maximal exercise ( $p$ $\leqslant 0.0001$ and $p=0.0003$, respectively). The mean maximal systolic blood pressure, however, was lower than the expected values for normal subjects (table 3). Patients on ACE inhibitors $(68 \mathrm{~mm} \mathrm{Hg})$ had a significantly lower diastolic blood pressure at rest compared to the patients without ACE inhibitors (76 $\mathrm{mm} \mathrm{Hg}, \mathrm{p}=0.003$ ). The same trend was found for systolic blood pressure at rest, and for diastolic and systolic blood pressure at maximal exercise, but no significant difference was shown.

\section{EJECTION FRACTION}

Normal resting values were found for both systemic right ventricular $(48 \%)$ and pulmonary left ventricular $(46 \%)$ ejection fractions (table 2 ). Systemic right ventricular ejection fraction only rose by $2 \%$ on exertion, less than the $5 \%$ or more expected in a normal population. Pulmonary left ventricular ejection fraction decreased by $2 \%$ on exertion (table 3). A multivariate linear regression model was used

Table 3 Exercise data (mean (SD)) in patients with CCTGA

\begin{tabular}{lll}
\hline Variable & $n$ & Values \\
\hline $\mathrm{Vo}_{2} \mathrm{max}(\mathrm{ml} / \mathrm{kg} / \mathrm{min})$ & 29 & $19.6(7.4)$ \\
$\mathrm{VO}_{2} \mathrm{max}(\mathrm{ml} / \mathrm{min})$ & 29 & $1374(530)$ \\
$\mathrm{SaO}_{2} \mathrm{max}(\%)$ & 24 & $92(8)$ \\
$\mathrm{HRmax}^{(\mathrm{bpm})}$ & 29 & $150(49)$ \\
Maximal systolic BP (mm Hg) & 27 & $153(19)$ \\
Maximal diastolic BP (mmHg) & 27 & $82(13)$ \\
Maximal MRVEF sys (\%) & 19 & $50(14)$ \\
Maximal MLVEF pulm (\%) & 14 & $44(20)$ \\
\hline
\end{tabular}

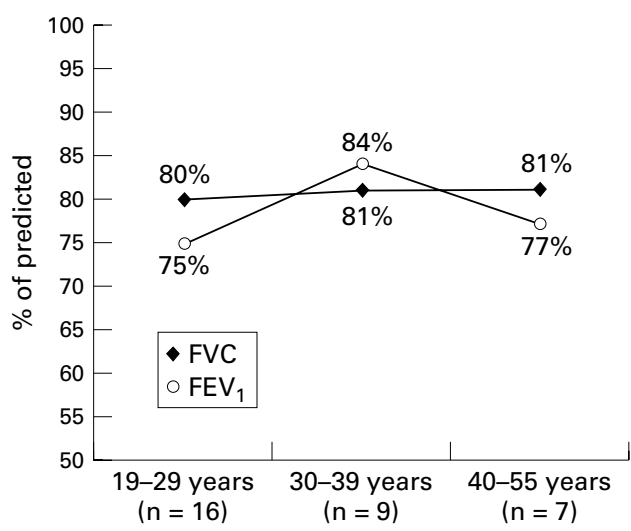

Figure 2 Percent of predicted values (12) in CCTGA patients with regard to forced expiratory capacity (FVC) and forced expiratory volume in one second (FEV). to examine the relation between sex, surgery, associated defects, ACE inhibitors, and outcome of maximal systemic right ventricular ejection fraction. Surgery turned out to have a negative effect on the systemic right ventricular ejection fraction. A $t$ test showed that patients without surgery achieved a significantly higher systemic right ventricular ejection fraction $(62 \%)$ on exertion than the surgery group $(46 \%)(p=0.033)$.

A similar multivariate linear regression model was used to examine the outcome of maximal pulmonary left ventricle ejection fraction. The results showed that patients treated with ACE inhibitors showed a significantly lower ejection fraction at maximal exercise $(34 \%)$ in the pulmonary left ventricle compared to the non-ACE group (58\%) $(p=0.014)$. In addition, the pulmonary left ventricular ejection fraction decreased for the ACE group from rest to maximal workload (37\% to $34 \%$ ), but increased for the non-ACE group (55\% to $58 \%$ ).

\section{LUNG FUNCTION}

Patients with CCTGA achieved significantly lower values of FVC $(p=0.005)$ and $\mathrm{FEV}_{1}$ ( $p=0.003$ ) compared to the predicted values. Patients without surgery (95\%) achieved predicted values of FVC more frequently than the surgery group $(79 \%, p=0.008)$. A similar trend was also shown for $\mathrm{FEV}_{1}(\mathrm{p}=0.087)$ (fig 2).

\section{Discussion}

Diminished aerobic capacity and lung function of adult patients with CCTGA when compared to predicted values for healthy individuals was shown in the present study. In addition, low values of maximal systolic blood pressure, maximal heart rate, and $\mathrm{SaO}_{2}$ at maximal exercise were found. A reduced increase in ejection fraction with exercise in the systemic right ventricle was displayed. Also a decrease in ejection fraction with exercise was demonstrated in the pulmonary left ventricle.

\section{AEROBIC CAPACITY}

In patients with CCTGA, an important issue has been the performance of the morphologic right ventricle when executing systemic pumping over decades. ${ }^{8}$ It has been suggested that systemic ventricular dysfunction in CCTGA is uncommon before the age of 35 years. ${ }^{814}$ 
However, resting values may be of limited interest as ventricular dysfunction often is first shown during exercise. ${ }^{15}$ As shown in fig 1, patients with CCTGA have severely diminished values of $\mathrm{VO}_{2} \mathrm{max}$ at all age groups. In addition, a significant fall off in $\mathrm{VO}_{2}$ max with age is apparent $(p=0.003)$. This may suggest a decrease in systemic right ventricular function with age. Hornung and colleagues have suggested a high prevalence of myocardial defects in unoperated patients with CCTGA with consequent abnormalities of region, wall motion and thickening, and impaired ventricular contractility ${ }^{16}$ Ischaemia and infarction may therefore be important contributors to ventricular failure. Alternatively, a left ventricular outflow tract obstruction, as in patients with conduits, or a dynamic left ventricular outflow tract obstruction because of exercise induced systolic anterior motion of the mitral valve, may also serve as a hypothesis for changes in ventricular performance with exercise.

The lower lung function values found in CCTGA patients compared to the predicted values may also have had an impact on patients' aerobic capacity. To support this suggestion the results from the multiple linear regression model showed that high FVC correlated with high $\mathrm{VO}_{2} \max (\mathrm{p}=0.002)$.

HEART RATE

The mean maximal heart rate in the present study was $79 \%$ of the predicted value, and the low value is in accordance with a previous report. ${ }^{8}$ This chronotropic incompetence may be an important factor that contributes to the diminished $\mathrm{VO}_{2} \max$; thus the HRmax could explain $64 \%$ of the increase in oxygen uptake $(\mathrm{ml} / \mathrm{kg} / \mathrm{min})$. A significant impairment of the chronotropic response has previously been shown to correlate with abnormally low $\mathrm{VO}_{2}$ max values. ${ }^{17}$ The regression model in the present study revealed a similar pattern, since the high HRmax values are correlated with a high $\mathrm{Vo}_{2} \mathrm{max}$. The fact that 10 patients were paced may also have influenced the results of HRmax. However, only five patients needed rate-responsiveness and it is therefore less likely this would have a major impact on the average values of HRmax.

\section{BLOOD PRESSURE}

A significant increase in both systolic and diastolic blood pressure was found with exercise; however, the maximal systolic blood pressure was lower than expected. This may support the assumption of an exercise induced deterioration of the systemic right ventricle, as the inability to increase blood pressure may influence the aerobic capacity. ${ }^{18}$

\section{EJECTION FRACTION}

Systemic right ventricle

As previously mentioned, there is an increase in systemic right ventricular ejection fraction with exercise which, however, is less than the expected 5\%. ${ }^{19}$ A deterioration of the systemic ventricle may to a greater extent influence aerobic capacity than the pulmonary ventricle, and may be the main reason for the diminished values of $\mathrm{VO}_{2}$ max shown in the present study. Parrish and colleagues suggested that for most patients, abnormality of ventricular function is not clinically important in childhood. ${ }^{19}$ The present data indicate that for adults, a deterioration of systemic right ventricular function has an impact on physical ability, in agreement with others. ${ }^{14}$

\section{Pulmonary left ventricle}

Previous papers have suggested normal function in the systemic ventricle, but an impaired pulmonary ventricular function. ${ }^{814}$ Others have reported normal ejection fraction in the pulmonary left ventricle, but no increase in the systemic right ventricle ejection fraction with exercise. ${ }^{15}$ The data in the present study show a small mean decrease of the ejection fraction in the pulmonary left ventricle. The explanation for the decrease in pulmonary ejection fraction is unclear. A hypothesis may be that pacing and arrhythmia could affect exercise intolerance in the CCTGA group; however, analyses show no differences in $\mathrm{VO}_{2}$ max between patients with pacemakers and patients in sinus rhythm. A possible explanation may be that the systemic atrioventricular valvar regurgitation may worsen during exercise, resulting in a relatively higher systemic ventricular ejection fraction, and thus raising pulmonary venous pressures and pulmonary capillary wedge pressures in turn. This would result in exercise intolerance as reported to occur with the systemic mitral valve in patients with mild rheumatic mitral valve disease at rest. ${ }^{20}$ In addition, the diastolic pressure overload of the morphological right ventricle may be at the expense of pulmonary left ventricular volume as the septum is bulging into the ventricle. This may result in impaired diastolic filling of the left ventricle as suggested in previous studies of ventricular interdependence, and a lower ejection fraction on the pulmonary side. ${ }^{21}$

The lower values found in patients on ACE inhibitors have a selection bias, as these patients have poorer ventricular function than patients without ACE. The fact that ejection fraction decreases with exercise in patients treated with ACE probably emphasises this. Unfortunately, there were no data available before and after the introduction of ACE inhibitors; thus the present data do not reveal the effect of ACE inhibitors in patients with CCTGA.

\section{MORTALITY}

Both early and late mortality in patients with CCTGA is quite high. In 1976, Bjarke and Kidd reported a $25 \%$ early paediatric mortality in patients with CCTGA including death during surgery. ${ }^{3}$ The results are based on data from the Hospital for Sick Children in Toronto, the same hospital as the patients in the present study attended before they were transferred to TCCCA. The treatment of these patients has improved since the 1970s, although the late mortality of $21 \%$ in our study showed that this still is a high risk group at all ages and should be followed with special attention. ${ }^{3}$ 
LIMITATIONS OF THE STUDY

This study is limited by the follow up constraints inherent in caring for adults with congenital heart disease. Many of our patients reside outside Toronto, and those that are well may be less interested in returning to our centre for cardiopulmonary testing. Our 41 of 84 patients may therefore represent a biased sample towards patients with more medical problems and poorer exercise capacity. There were too few patients with measurements of ejection fraction at maximal exercise to include these variables in a linear regression model with $\mathrm{VO}_{2}$ max as the dependent variable. A continuous follow up of these patients with ejection fraction measurements would give additional information for future research. The limited number of patients available implies that failure to regret a hypothesis does not necessarily imply equality.

CONCLUSION

Diminished values of heart rate, $\mathrm{FEV}_{1}, \mathrm{FVC}$, and systolic blood pressure compared to the predicted values may contribute to the reduced $\mathrm{VO}_{2}$ max found in patients with CCTGA. In addition, a limited increase in systemic right ventricular ejection fraction and a decrease in pulmonary left ventricle contractility suggest a dysfunction of both ventricles. A larger prospective study should be carried out in order to confirm the present data.

PMF has been supported by the Norwegian Association for Children with Congenital Heart Disorder (FFHB), the Norwegian Lung and Heart Association, and by the National Foundation of Public Health in Norway. GV has been supported by the National Heart Research Fund, UK.

1 Von Rokitansky K. Die Defecte der Scheidewande des Herzens. In: Anonymous Pathologische-anatomische Abhandlung. Vienna, 1875:83-6.

2 Webb GD, McLaughlin PR, Gow RM, et al. Transposition complexes. Cardiol Clin 1993;11:651-64.

3 Bjarke BB, Kidd BS. Congenitally corrected transposition of the great arteries. A clinical study of 101 cases. Acta Paediatr 1976;65:153-60.

4 Schiebler GL, Edwards JE, Burchell HB, et al. Congenital corrected transposition of the great vessels: a study of 33 cases. Pediatrics 1961;27:851-88.
5 Rotem CE, Hultgren HN. Corrected transposition of the great vessels without associated defects. Am Heart f 1965; 0:305-18.

6 Puley G, Siu S, Connelly M, et al. Arrhythmia and survival in patients $>18$ years of age after the Mustard procedure for complete transposition of the great arteries. Am $\mathcal{F}$ Cardiol 1999;83:1080-4.

7 Masden RR, Franch RH. Isolated congenitally corrected transposition of the great arteries. In: Hurst JW, ed. The heart, 3 ed. New York: McGraw-Hill, 1980:59-83.

8 Benson LN, Burns R, Schwaiger M, et al. Radionuclide angiographic evaluation of ventricular function in isolated congenitally corrected transposition of the great arteries. Am 7 Cardiol 1986;58:319-24.

9 Dimas AP, Moodie DS, Sterba R, et al. Long-term function of the morphologic right ventricle in adult patients with corrected transposition of the great arteries. Am Heart $\mathcal{F}$ 1989;118:526-30.

10 James FW, Blomqvist CG, Freed MD, et al. Standards for exercise testing in the pediatric age group. American Heart Association council on cardiovascular disease in the young. Ad hoc committee on exercise testing. Circulation 1982; 66:1377A-97A.

11 American Thoracic Society. Lung function testing: selection of reference values and interpretive strategies. Am Rev Respir Dis 1991;99:408-15.

12 Percentile distributions of anthropometric and fitness variables by age (Canadians aged 10 and older). 1999. Canadian Fitness and Lifestyle Research Institute. Campbell Survey on Well-Being in Canada (1988).

13 Jones NL. The interpretation of stage 1 exercise test results. In: Trumbold C, ed. Clinical exercise testing. Philadelphia: WB Saunders, 1988:158-85.

14 Graham TPJ, Parrish MD, Boucek RJJ, et al. Assessment of ventricular size and function in congenitally corrected transposition of the great arteries. Am f Cardiol 1983;51: 244-51.

15 Peterson RJ, Franch RH, Fajman WA, et al. Comparison of cardiac function in surgically corrected and congenitally corrected transposition of the great arteries. 7 Thorac Cardiovasc Surg 1988;96:227-36.

16 Hornung TS, Bernard EJ, Jaeggi ET, et al. Myocardial perfusion defects and associated systemic ventricular dysfunction in congenitally corrected transposition of the great arteries. Heart 1998;80:322-6.

17 Schulze-Neick IM, Wessel HU, Paul MH. Heart rate and oxygen uptake response to exercise in children with low peak exercise heart rate. Eur F Pediatr 1992; 151:160-6.

18 Astrand PO, Rodahl K. Textbook of work physiology, 3 ed. New York: McGraw-Hill, 1986.

19 Parrish MD, Graham TPJ, Bender HW, et al. Radionuclide angiographic evaluation of right and left ventricular function during exercise after repair of transposition of the great arteries. Comparison with normal subjects and patients with congenitally corrected transposition. Circulapatients with congenitall
tion $1983 ; 67: 178-83$.

20 Tischler MD, Battle RW, Saha M, et al. Observations suggesting a high incidence of exercise-induced severe mitral regurgitation in patients with mild rheumatic mitral valve disease at rest. f Am Coll Cardiol 1995;25:128-33.

21 Santamore WP, Dell'Italia LJ. Ventricular interdependence: significant left ventricular contributions to right ventricular systolic function. Prog Cardiovasc Dis 1998;40:289-308. 